\title{
Weight Bias Internalization: Semantic Differential Measurement and Treatment Implications
}

\author{
Raymond C. Hawkins II \\ Fielding Graduate University; The University of Texas at Austin, Austin, USA
}

\begin{abstract}
Stereotypic attributions about "overweight people" (called weight stigma or bias) and "slender people" were elicited through semantic differential procedures and compared with self-attributions on the same bipolar personality trait scales. More negative personality traits were attributed to overweight people than to slender people and self-descriptions. Overweight subjects attributed negative traits to "overweight people" but did not self-endorse these negative personality characteristics. Internalization of weight bias was associated with a negative body-image and binge-eating, irrespective of actual body weight. Although these correlations are consistent with those of Carels et al. (2013), re-analysis of our findings using Grice's (2011) “Observation Oriented Modeling” method showed a low percentage of correct classification of participants' self-perceived weight stigma based on negative body image. Caution is urged in inferring psychological maladjustments of persons internalizing weight bias in the absence of research showing that these effects hold for actual persons rather than statistical parameters.
\end{abstract}

Keywords: weight bias internalization, obesity stigma, negative personality traits, weight loss maintenance

\section{Introduction}

Biopsychosocial theories of obesity and diet-consciousness assume the existence of a negative stereotype for overweight and the social desirability of thinness (e.g., Hawkins \& Clement, 1984; Rodin, 1977). Negative personality traits are attributed by both females and males to the overweight (endomorphic) body build (e.g., Carels et al., 2011; Crandall, 1994; Harris, Harris, \& Bochner, 1982). More recently this negative stereotype for obesity has been called weight stigma or weight bias (Puhl \& Heuer, 2009; Puhl \& Suh, 2015). Given the ubiquity of the negative stereotype for this weight bias, it is a question of considerable theoretical and practical interest as to the psychological mechanism through which a generalized cultural expectancy regarding the overweight stereotype becomes personally relevant, i.e., when these negative trait descriptors are self-attributed. Several researchers have recently been studying this internalization of the weight bias/stigma (e.g., Carels et al., 2011, 2013; Durso \& Latner, 2008; Gordijn, 2010; Lillis, Luoma, Levin, \& Hayes, 2010; Major, Hunger, Bunyan, \& Miller, 2014; Puhl, Moss-Racusin, \& Schwartz, 2007), how to ameliorate its impact (e.g., Danielsdottir, O'Brien, $\&$ Ciao, 2011), and its apparent refractoriness to change as a function of weight-loss treatment (Carels et al., 2009, 2014; Levy \& Pilver, 2012).

Carels et al. (2013) described what they termed a novel finding that although overweight clients participating in a weight loss program endorsed the negative stereotype of obesity involving negative personality

Raymond C. Hawkins II, Ph.D., ABPP, Core Faculty, Fielding Graduate University; Clinical Assistant Professor, Psychology Department, The University of Texas at Austin. 
traits (e.g., lazy, undisciplined), they did not self-attribute these undesirable traits. These self-attributed negative traits were also significantly associated with a measure of internalized weight bias, and with self-report measures of depression, binge eating, and negative body image. Examining discrepancy scores between obese negative stereotype trait ratings and self-trait ratings, they found that the greater the difference (i.e., higher the obese negative stereotype ratings and the lower the negative self-trait ratings) the lower the depression, binge eating, and body dissatisfaction. Finally, they hypothesized that those clients who did self-attribute these negative trait characteristics (i.e., those who internalized the weight stigma/bias) rather than the positive traits of normal weight persons would be less successful in losing weight and sustaining their weight loss. The Carels et al. (2013) study, if replicated, has important implications for the treatment of obesity and eating disorders.

The purposes of the present study were: (1) to describe a semantic differential procedure used in an unpublished study (Doell \& Hawkins, 1981) to demonstrate the existence of a negative stenotype for overweight people and a set of more positive stereotypic attributions about "slender people"; (2) to examine the relationship between negative stereotypic attributions about "overweight people" to self-attributions using the same bipolar adjectives (i.e., to determine whether subjects who themselves are overweight would self-endorse-negative trait terms attributed to overweight people); (3) to study the relationship between these stereotypic attributions and personality self-descriptions to perceptions of physical and behavioral characteristics (e.g., body image, eating "restraint", and binge eating tendencies); (4) to investigate whether students participating in a weight control program self-attribute more of the negative trait terms of the overweight stereotype than do equivalently overweight subjects not in treatment; and (5) to reanalyze these data using Grice's (2011) "Observation Oriented Modeling" to determine whether these parametric effects are verified by the percentage of correct classifications of actual participants as a function of the hypothesized causal relationships.

\section{Method}

\section{Samples}

Three samples of college undergraduates of varying body weights were drawn from psychology classes. Students volunteered for this research for extra credit. The first sample contained 188 females and 85 males, the second sample had 124 females and 46 males, and the third sample contained 44 females and 20 males. Body weight percentages were calculated as a deviation percentage from the "desirable" weight for males and females with a medium body frame (Metropolitan Life Insurance Company, 1959). Subjects were considered overweight if their self-reported body weight was at least twenty percent greater than the desirable weight for their stated heights. A fourth sample of 15 overweight clients (11 females, 4 males) (mean excess weight of 25\%) from a behavioral weight control program also participated in this study. For the data re-analysis using Grice's (2011) "OOM" method, the first sample and third sample were used. Informed consent was obtained from all individual participants included in the study.

\section{Procedure}

The semantic differential procedure (Osgood, Suci, \& Tannenbaum, 1957) was used to elicit the meanings of three concepts: "myself", "overweight people" (sex and degree of overweight unspecified), and "slender people" (sex and weight unspecified). The same 44 scales comprised the semantic differential used to evaluate each of the concepts. Each 5-point scale was anchored on the extremes with bipolar adjectives and had a neutral point. Sample adjective pairs included: independent vs. dependent; active vs. passive; healthy vs. sick; graceful 
vs. awkward) ${ }^{1}$. The third sample completed these 44 scales for four concepts: "myself", "overweight people" (of same sex as subject, 15 pounds overweight), "slender people" (of same sex as subject, 10 pounds underweight), and "obese people" (of same sex as subject, 35 pounds overweight). The Negative Self-Image Scale ${ }^{2}$ was administered to the first and second samples. The Binge Scale (Hawkins \& Clement, 1980) and the Restraint Scale (Herman \& Polivy, 1975) were obtained from subjects in the second sample.

\section{Results}

For both the first and second samples, Oneway ANOVAs on the 44 semantic differential scales revealed that the meanings of the concepts "myself", "overweight people", and "slender people" differed significantly $(p<$ 0.001). The concept "overweight people" was judged most extreme in the socially undesirable direction for 38 of the 44 scale means. To simplify analysis of concept differences, 25 bipolar adjective scales were chosen for calculation of a summary score for the "overweight people" concept, yielding a Cronbach's alpha of 0.85 . The decision rule for selecting this subset of adjectives was that the mean rating on each for the "overweight people" concept fell on the opposite side of neutrality from the ratings from the corresponding adjectives for the concepts "slender people" and "myself". For all 25 bipolar adjectives, the mean ratings for "overweight people" fell on the socially undesirable side of neutrality, in contrast to the socially desirable ratings for the other two concepts. The means and standard deviations for the semantic differential concept scores for male and female, normal weight and overweight subjects from the three samples (plus the clinic sample) are presented in Table 1. The higher the average score, the more negative (socially undesirable) the stereotypical attribution. The concept "overweight people" was rated more negatively by all groups relative to the ratings for the other concepts.

ANOVAs with two between-subject factors (sex, body weight) were performed on each of the three concept scores. For the first sample, for the concept "overweight people" there was a statistically significant main effect for body weight, $F(1,246)=11.74, p<0.001$, with the overweight students (mean excess weight $=28 \%, S D=$ 17.0) evaluating the concept less negatively than did the normal weight students. For the concept "slender people", males' scores were significantly more negative (higher) than those of females, $F(1,246)=5.67, p<0.05$. There were no sex or weight related differences for the concept "myself". For the second sample, ANOVAs performed on each of the concept scores revealed no statistically significant main effects.

Table 1 shows that obese females in the classroom sample and in the clinic sample rated the concept "myself" almost as favorably as did their normal weight counterparts, suggesting the operation of a self-protective bias. This interpretation should be considered within the context of findings with the other measures. Overweight students in both samples were more critical of their physical appearance relative to the normal weight students, and the former reported more eating "restraint" and stronger binge eating tendencies. Regardless of the subjects' actual body weights, individuals who thought they were fat (i.e., had a negative body image) reported a more negative "self" concept (Table 2).

Table 3 shows the data from a replication of this semantic differential study (the third sample), in which the evaluative task was refined. The target concepts "overweight people" and "slender people" were operationally defined as to gender and pounds, and a more extreme concept "obese people" was added. The results were that the concepts "overweight people" and "obese people" were rated more negatively relative to the concepts

\footnotetext{
${ }^{1}$ For copies of the semantic differential scales used in this research please e-mail the author (rhawkins@utexas.edu).

${ }^{2}$ Nash, personal communication, 1977. For further information about the Negative Self-Image Scale, see Hawkins \& Clement (1980), as cited in the references.
} 
"myself" and "slender people". While the overweight students' attributions for the concepts "overweight people" and "obese people" were as negative as those of the normal weight students, once again the former's scores on the concept "myself" were nearly as favorable as were the latter's, thus replicating the effect found in the earlier studies.

Table 1

Means and Standard Deviations for the Semantic Differential Concept Scores for Male and Female, Normal Weight and Overweight Subjects in Several Samples

\begin{tabular}{|c|c|c|c|c|c|c|c|}
\hline \multirow[t]{3}{*}{ Sex } & \multirow[t]{3}{*}{ Weight } & \multicolumn{2}{|c|}{ Myself } & \multicolumn{2}{|c|}{ Overweight } & \multirow{2}{*}{\multicolumn{2}{|c|}{$\frac{\text { Slender }}{S D}$}} \\
\hline & & $M$ & $S D$ & $M$ & $S D$ & & \\
\hline & & Initial & Sample & & & & \\
\hline Males $^{\mathrm{a}}$ & Normal & 2.31 & 0.47 & 3.49 & 0.45 & 2.57 & 0.39 \\
\hline Females $^{\mathrm{b}}$ & Normal & 2.33 & 0.39 & 3.46 & 0.42 & 2.44 & 0.40 \\
\hline \multicolumn{8}{|c|}{ Second Sample } \\
\hline Males $^{\mathrm{c}}$ & Normal & 2.34 & 0.44 & 3.39 & 0.49 & 2.45 & 0.34 \\
\hline Females $^{\mathrm{d}}$ & Normal & 2.35 & 0.43 & 3.53 & 0.43 & 2.38 & 0.42 \\
\hline \multicolumn{8}{|c|}{ Third Sample } \\
\hline Females $^{\mathrm{e}}$ & Clinic Obese & 2.54 & 0.36 & 3.95 & 0.40 & 1.87 & 0.34 \\
\hline Females $^{\mathrm{f}}$ & Classroom Obese & 2.38 & 0.44 & 3.50 & 0.32 & 2.48 & 0.46 \\
\hline
\end{tabular}

Notes. ${ }^{\mathrm{a}} n=86 ;{ }^{\mathrm{b}} n=188 ;{ }^{\mathrm{c}} n=46 ;{ }^{\mathrm{d}} n=124 ;{ }^{\mathrm{e}} n=11 ;{ }^{\mathrm{f}} n=1$.

Table 2

Correlations among Concept Measures, Negative Self-Image (NSI), Restraint (EST), Binge Scale (BST), and Excess Weight Percentage (WGTPERC) for the Second Sample $(n=117)$

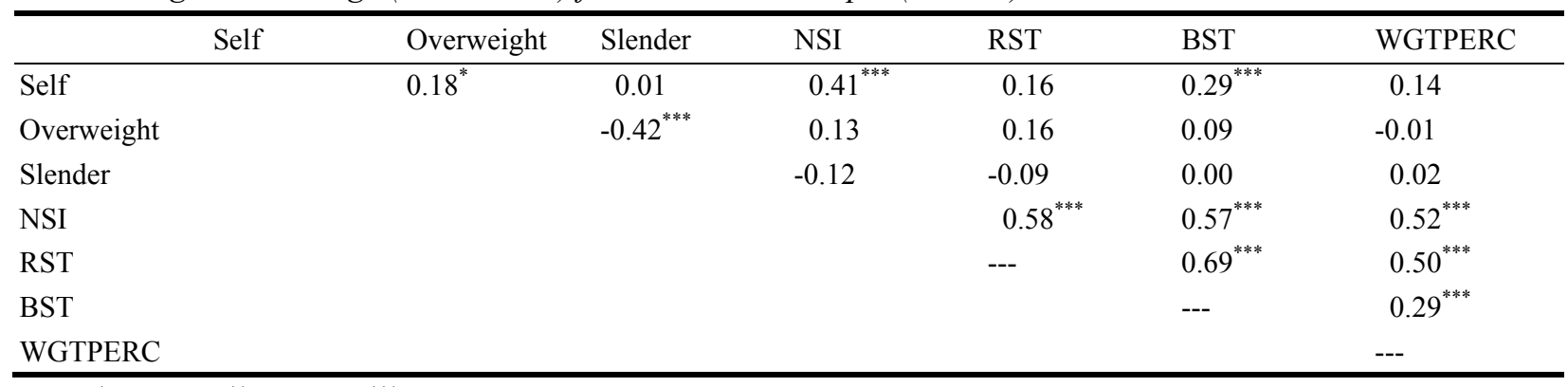

Notes. ${ }^{*} p<0.05 ;{ }^{* *} p<0.01 ;{ }^{* * *} p<0.001$; These instruments are scored in the negative (socially undesirable) direction.

Table 3

Means and Standard Deviations for the Semantic Differential Concept Scores for Male and Female, Normal Weight and Overweight Subjects in the Second Replication Sample, in Which the Sex and Weight of the Target Person Were Specified

\begin{tabular}{llllllllll}
\hline Sex & Weight & \multicolumn{3}{c}{ "Myself" } & \multicolumn{2}{c}{ "Overweight people" } & \multicolumn{2}{c}{ "Obese people" } & \multicolumn{2}{c}{ "Slender people" } \\
\hline & & $M$ & $S D$ & $M$ & $S D$ & $M$ & $S D$ & $M$ & $S D$ \\
\hline Males $^{\mathrm{a}}$ & Normal & 2.15 & 0.30 & 3.02 & 0.41 & 3.62 & 0.14 & 2.37 & 0.34 \\
Females $^{\mathrm{b}}$ & Normal & 2.15 & 0.43 & 3.20 & 0.26 & 3.57 & 0.40 & 2.38 & 0.50 \\
Males $^{\mathrm{c}}$ & Overwgt. & 2.05 & 0.35 & 3.07 & 0.45 & 3.54 & 0.62 & 2.58 & 0.41 \\
Females $^{\mathrm{d}}$ & Overwgt. & 2.54 & 0.57 & 3.24 & 0.37 & 3.58 & 0.34 & 2.52 & 0.41 \\
\hline
\end{tabular}

Notes. ${ }^{\mathrm{a}} n=8 ;{ }^{\mathrm{b}} n=23 ;{ }^{\mathrm{c}} n=12 ;{ }^{\mathrm{d}} n=21$. 
Weight loss outcomes at the end of group treatment were available for 14 of the 15 clinic overweight clients from whom semantic differential ratings of "overweight people", "slender people", and "myself" were obtained at the start of treatment. For seven of these clinic overweight clients, follow-up data two to four years later were also available. Spearman's rho non-parametric correlations showed that although internalization of the overweight stereotype bias was not significantly associated with weight loss during treatment ( $\mathrm{rho}=0.266, p=$ 0.358 , 2-tailed test), it was significantly positively correlated with continued weight loss at follow-up (rho $=$ $0.929, p=0.003,2$-tailed test).

Finally, reanalysis of these semantic differential data was done using Grice's (2011) Observation Oriented Modeling (OOM). However, these OOM non-parametric analyses revealed that only 117 of 233 observations of the negative body image effect (50.21\%) from the first student sample could be correctly classified based on conforming to the hypothesized cause of the self-trait ratings ("myself"), yielding a "c" (chance) value of 0.11, which is likely no greater than chance. Crossing gender with self-trait ratings did not improve the percent of negative body image correctly classified. Discrepancy analyses of the internalized negative trait ratings (i.e., "myself"- "overweight people" ratings) correctly classified only 48 of 233 (20.43\%) observations for negative self image ( $c$-value of 0.76 ), and "thin bias" trait ratings ("myself"- "slender people" ratings) correctly classified 84 of 233 observations (36.05\%) for negative self-image ( $c$-value of 0.04). Crossing gender with these discrepancy score internalized negative trait ratings or thin bias ratings did not improve the percent correctly classified. Similarly, OOM re-analyses for the third sample $(n=64)$ produced a correct classification of just $56.25 \%$ ( 29 out of 64 cases) of the negative body self-image effect based on conforming to the hypothesized cause of the self-trait ratings ("myself")(c-value of 0.11 ), and the discrepancy analyses (i.e., "overweight internalized bias", or "myself" — "overweight people"; "obese internalized bias", or "myself" — "obese people"; "thin bias", or "myself"- "slender people") yielded correct classification percentages of less than $32 \%$. Crossing gender or overweight percentage with these discrepancy scores did not improve the correct classification percentages.

\section{Discussion}

In these samples, college students of varying body weights consistently attributed more socially undesirable personality trait characteristics to "overweight people" and "obese people" relative to "slender people" and "myself". More important, there was no relationship between the negative stereotypic attributions for overweight and self-attributions on the same bipolar adjective scales. Scores on the concept "myself" were as favorable for the overweight students as for their normal weight peers. These findings, in part originally reported by Doell and Hawkins (1981), are consistent with the results of Carels et al. (2013) in his small sample of 68 obese clients seeking weight loss treatment.

Also consistent with the findings of Carels et al. (2013), overweight college females participating in a weight control program did not self-attribute more of the trait terms of the overweight negative stereotype (i.e., internalized weight bias traits), relative to equivalently overweight college women not in treatment. Women seeking assistance for weight loss described their personality characteristics as positively as did the other subjects in these samples. However, examination of weight loss outcomes for seven clinic treated clients showed that continued weight loss during follow-up was significantly positively correlated with internalization of the overweight negative personality bias. This finding supports Carels et al.'s (2013) claim that internalizing the negative personality traits of the obese stereotype may predict outcomes in weight loss treatment, but the 
direction of the effect was opposite to their prediction. Implications of the clinical sample's more negative evaluations of "overweight people" and more positive attributions about "slender people" requires further research.

An important caveat is that when these data were reanalyzed using Grice's (2011) "Observation Oriented Modeling", negative body self-image as an effect could be correctly classified based on conforming to the hypothesized cause of the negative self-trait ratings for only about $50 \%$ of the cases. Additional OOM analyses examining weight bias, internalized weight bias, and thin bias yielded even lower correct classification rates. In conclusion, unless Carels et al. (2013) or other researchers can show that these effects are robust at the person level of analysis (i.e., that internalization of the obese negative personality stereotype predicts weight loss through treatment and its maintenance at follow-up), which is the goal of predicting outcomes in bariatric behavioral medicine, we will need to remain cautious about making these claims.

\section{References}

Carels, R. A., Young, K. M., Wott, C. B., .., \& Clayton, A. M. (2009). Weight bias and weight loss treatment outcomes in treatment-seeking adults. Annals of Behavioral Medicine, 37(3), 350-355.

Carels, R. A., Hinman, N., Koball, A., ..., \& Young, K. M. (2011). The self-protective nature of implicit identity and its relationship to weight bias and short-term weight loss. Obesity Facts, 4, 278-283.

Carels, R. A., Burmeister, J., Oehlhof, M. W., ..., \& Ashrafioun, L. (2013). Internalized weight bias: Ratings of the self, normal weight, and obese individuals and psychological maladjustment. Journal of Behavioral Medicine, 36(1), 89-64.

Carels, R. A., Hinman, N. G., Hoffman, D. A., ..., \& Ashrafioun, L. (2014). Implicit bias about weight and weight loss treatment outcomes. Eating Behavior, 15(4), 648-653.

Crandall, C. S. (1994). Prejudice against fat people: Ideology and self-interest. Journal of Personality and Social Psychology, 66(5), 882-894.

Danielsdottir, S., O’Brien, K., \& Ciao, A. (2010). Anti-fat prejudice reduction: A review of published research. Obesity Facts, 3 , 47-58.

Doell, S. R., \& Hawkins, R. C. II. (1981). Obesity, slimness, and self-protective bias: A semantic differential analysis. (Unpublished paper presented at the American Psychological Association, Los Angeles, CA).

Durso, L. E., \& Latner, J. D. (2008). Understanding self-directed stigma: Development of the Weight Bias Internalization Scale. Obesity, 16, S80-S86.

Gordijn, E. H. (2010). When thinking that you are fat makes you feel worthless: Activation and application of meta-stereotypes when appearance matters. Social Cognition, 28(1), 20-39.

Grice, J. (2011). Observation oriented modeling: Analysis of cause in the behavioral sciences. Burlington, M.A.: Academic Press.

Harris, M. B., Harris, R. J., \& Bochner, S. (1982). Fat, four-eyed, and female: Stereotypes of obesity, glasses, and gender. Journal of Applied Social Psychology, 12(6), 503-516.

Hawkins, R. C. II, \& Clement, P. F. (1980). Development and construct validation of a self-report measure of binge eating tendencies. Addictive Behaviors, 5, 219-226.

Hawkins, R. C. II, \& Clement, P. F. (1984). Binge eating: Measurement problems and a conceptual model. In R. C. Hawkins II, W. J. Fremouw, \& P. F. Clement (Eds.), The binge-purge syndrome: Diagnosis, treatment, and research (pp. 229-253). New York: Springer.

Herman, C. P., \& Polivy, J. (1975). Anxiety, restraint, and eating behavior. Journal of Abnormal Psychology, 84, 656-672.

Levy, B. R., \& Pilver, C. E. (2012). Residual stigma: Psychological distress among the formerly overweight. Social Science Medicine, 75(2), 297-299.

Lillis, J., Luoma, J. B., Levin, M. E., \& Hayes, S. C. (2010). Measuring weight stigma: The Weight Self-stigma Questionnaire. Obesity, 18, 971-976.

Major, B., Hunger, J. M., Bunyan, D. P., \& Miller, C. T. (2014). The ironic effects of weight stigma. Journal of Experimental Social Psychology, 51, 74-80.

Metropolitan Life Insurance Company of New York. (1959). New weight standards for males and females. Statistical Bulletin, 40, 2-3. 
Osgood, C. E., Suci, G. J., \& Tannenbaum, P. M. (1957). The measurement of meaning. Chicago: University of Illinois Press. Puhl, R. M., \& Heuer, C. A. (2009). The stigma of obesity: A review and update. Obesity, 17, 941-964.

Puhl, R. M., Moss-Racusin, C. A., \& Schwartz, M. B. (2007). Internalization of weight bias: Implications for binge eating and emotional well-being. Obesity, 15, 19-23.

Puhl, R. M., \& Suh, Y. (2015). Stigma and eating and weight disorders. Current Psychiatry Reports, 17, 10.

Rodin, J. (1977). Research on eating behavior and obesity: Where does it fit in personality and social psychology? Personality and Social Psychology Bulletin, 3, 333-355. 